\title{
DESAFÍOS DIDÁCTICOS DEL SABER PEDAGÓGICO DEL DOCENTE UNIVERSITARIO NOVEL CHILENO
}

\author{
Alejandro Antonio Villalobos-Claveria \\ Yenia Marcela Melo-HermosillaII \\ I Universidad de Concepción (UdeC), Concepción, (Bío-Bío), Chile; avillalo@udec.cl \\ "I Universidad de Concepción (UdeC), Concepción, (Bío-Bío), Chile; ymelo@udec.cl
}

\begin{abstract}
Resumen
Este estudio proporciona un análisis de la transferencia didáctica del profesor universitario novel y busca caracterizar el camino de su transformación en docente. La matriz metodológica de la investigación fue un estudio de caso en tres universidades regionales chilenas. Los datos provienen de la aplicación de una entrevista en profundidad y el análisis del currículo vitae del grupo consultado. Los resultados revelan elementos propios de la transposición didáctica en la construcción de saberes, donde convergen factores subjetivos, disciplinarios, institucionales y experiencias prácticas del profesor novel. Inicialmente se reproducen patrones de formación, los cuales son cuestionados frente a situaciones complejas. Interesa destacar que este conocimiento se construye como un diálogo de saberes: ciencia y pedagogía.
\end{abstract}

ENSEÑANZA SUPERIOR • DIDÁCTICA • UNIVERSIDADES • PROFESOR

\section{DESAFIOS DIDÁTICOS DO SABER PEDAGÓGICO DO PROFESSOR UNIVERSITÁRIO CHILENO PRINCIPIANTE}

\section{Resumo}

Este estudo nos oferece uma análise da transferência didática do professor universitário principiante e pretende caracterizar o caminho da sua transformação em docente. A matriz metodológica da pesquisa foi um estudo de caso em três universidades regionais chilenas. Os dados provêm da aplicação de uma entrevista em profundidade e da análise do curriculum vitae do grupo consultado. Os resultados revelam elementos próprios da transposição didática na construção de saberes, na qual convergem fatores subjetivos, disciplinares, institucionais e experiências práticas do professor principiante. Inicialmente se produzem padrões de formação, que são questionados perante situações complexas. Interessa destacar que esse conhecimento é construído como um diálogo de saberes: ciência e pedagogia.

ENSINO SUPERIOR • DIDÁTICA • UNIVERSIDADES • PROFESSOR 


\section{DIDACTICAL CHALLENGES OF THE PEDAGOGICAL KNOWLEDGE IN CHILEAN UNIVERSITY NEW TEACHER}

\section{Abstract}

This study provides an analysis of the didactic transfer of the novice university professor and seeks to characterize the path of his transformation into a teacher. The methodological matrix of the research was a case study in three regional Chilean universities. The data come from the application of an indepth interview and the analysis of the curriculum vitae of the group consulted. The results reveal elements of the didactic transposition in the construction of knowledge, where subjective, disciplinary, institutional factors and practical experiences of the new teacher converge. Initially, training patterns are reproduced, which are questioned in complex situations. It is interesting to note that this knowledge is constructed as a dialogue of knowledge: science and pedagogy.

HIGHER EDUCATION • TEACHING • UNIVERSITIES • TEACHER

\section{DÉFIS DIDACTIQUES DU SAVOIR PÉDAGOGIQUE DES PROFESSEURS UNIVERSITAIRES DÉBUTANTS AU CHILI}

\section{Résumé}

Cette étude propose une analyse du transfert didactique des professeurs universitaires débutants et cherche à caractériser leur parcours de transformation en enseignants. La base méthodologique de la recherche a consisté en une étude de cas au sein de trois universités régionales chiliennes. Les données ont été recueillies au cours d'un entretien approfondi ainsi que par l'analyse du curriculum vitae des participants. Les résultats ont mis en évidence des éléments propres à la transposition didactique dans l'élaboration des savoirs, où convergent des facteurs subjectifs, disciplinaires, institutionnels et des expériences pratiques des professeurs débutants. Les modèles de formation initialement produits sont remis en question face à des situations complexes. Il est intéressant de souligner que cette connaissance se construit comme un dialogue des savoirs : entre la science et la pédagogie.

ENSEIGNEMENT SUPÉRIEUR • DIDACTIQUE • UNIVERSITÉS • ENSEIGNANT 
PARTIR DE LA DECLARACIÓN DE BOLONIA Y EL PROYECTO TUNING EN AMÉRICA LATINA Y particularmente en Chile, se ha tratado de ofrecer autonomía al estudiante universitario que aprende una carrera profesional, lo cual ha provocado nuevos desafíos y nuevas problemáticas en el trabajo del profesorado de la educación superior chilena (Tuning América Latina, 2004; Brunner, 2015).

En este contexto, se puede afirmar que la docencia universitaria está bajo crecientes exigencias y demandas de calidad que provienen de esta sociedad del conocimiento (Brunner, 2015), como también del tipo de estudiante que ingresa al sistema de educación superior (Isaurralde, 2016; Walker, 2016).

El profesor universitario, cuando asume su condición de docente, enfrenta una disyuntiva: aferrarse al pasado o enfrentar su presente-futuro. Este docente y principalmente el profesor novel, viven una situación de desconcierto, pues a los tradicionales roles de profesor e investigador se ha unido otro, como gestor (facilitador) del aprendizaje estudiantil (Medina \& Jarauta, 2013; Rodríguez Torres et al., 2020; Tardif, 2004).

Al examinar la literatura especializada sobre pedagogía universitaria se reconoce una abundante investigación sobre diferentes facetas del trabajo del docente de educación superior, ya sea sobre sus creencias, estilos docentes, instrumentos de evaluación, competencias docentes, saberes pedagógicos, identidad profesional, desempeño competente, capacitación pedagógica (Bolívar \& Caballero, 2015; Fernández Lamarra \& Pérez Centeno, 2011; García-Prieto et al., 2019; Medina \& Jarauta, 2013; Villalobos-Claveria \& Melo-Hermosilla, 2019).

Sin embargo, solo en los últimos años, la voz de profesores nóveles comienza a ser reconocida como una expresión válida dentro de la comunidad académica (Guzmán, 2012; Porto Castro \& Mosteiro García, 2016; Rodríguez Torres et al., 2020), exceptuando algunos casos como el de Feixas (2002), quien analizaba problemáticas identificadas por este colectivo en contextos universitarios. Hasta ese momento, se esperaba que el profesor principiante cumpliera las exigencias de la carrera académica para ser reconocido como un par por sus colegas expertos.

Lo anterior permite afirmar que es necesario desarrollar investigaciones sobre el nuevo profesorado que se incorpora a la enseñanza universitaria y la forma que utiliza para generar conocimiento didáctico que permita responder a los desafios institucionales por una formación profesional superior de calidad (Guerra \& Montenegro, 2017). Por lo tanto, la problemática que aborda este estudio es la transformación de los conocimientos disciplinarios que posee un experto (investigador, doctor), cuando desarrolla su quehacer docente cotidiano, por medio de una gestión del conocimiento que le permite asumir una nueva función profesional, como profesor universitario. Tesis que asume este trabajo y que es un verdadero supuesto investigativo para su operacionalización metodológica.

Una primera perspectiva interesante de utilizar en la comprensión del trabajo del profesor novel es la mirada del "Enfoque Académico de la Enseñanza y el Aprendizaje", cuya sigla en inglés es SoTL ("Scholarship of Teaching and Learning"), que permite entender el ejercicio docente universitario y, a la vez, puede ser un basamento para configurar los principios fundantes de su futura profesionalización (Chocarro de Luis et al., 2013; Fanghanel et al., 2016; Albergaria-Almeida, 2010).

El SoTL aspira a ser una meta-teoría capaz de dar una visión global de la docencia universitaria y, al mismo tiempo, utiliza técnicas propias de la investigación educacional, tales como: entrevistas, cuestionarios, observación participante, encuestas, grupos focales, etc. y procedimientos asociados a la reflexión y análisis, triangulación de resultados, investigación cuasi experimental, entre otros (Fanghanel et al., 2016; Hutchings \& Shulman, 1999). 
Necesitamos reflexionar sistemáticamente sobre la evidencia del aprendizaje de nuestros propios estudiantes, nuestras propias clases y disciplinas. Necesitamos basarnos en la investigación genérica, pero cuidadosamente situarla dentro de nuestras disciplinas. Entonces necesitamos monitorear el éxito o no de nuestros esfuerzos para mejorar el aprendizaje de nuestros estudiantes y luego comunicar los resultados de esos esfuerzos a nuestros colegas. La beca de enseñar y aprender en esta perspectiva no es la investigación en el sentido tradicional; es una actividad de orientación para ser realizada de manera colegiada y cada vez más junto con la investigación tradicional dentro de las disciplinas. . . Para ser profesores eruditos, los académicos deben utilizar el mismo tipo de procesos de pensamiento en su enseñanza, que aplican a su investigación. ${ }^{1}$ (Albergaria-Almeida, 2010, p. 149, traducción propia).

La perspectiva de SoTL se ha extendido en países de habla inglesa y es poco conocida en países hispanoparlantes y algunos países europeos como Italia o Francia. No obstante, en casi toda Europa y Latinoamérica, se advierte la existencia de grupos y comunidades docentes universitarios de todas las disciplinas que buscan indagar, investigar y presentar resultados sobre aspectos que ayudan a mejorar y articular los procesos de enseñanza-aprendizaje en la docencia universitaria, es decir, surgen comunidades académicas motivadas por hacer un análisis científico de su práctica pedagógica y, por ende, avanzar en el conocimiento de mejores prácticas docentes que fomenten el aprendizaje estudiantil en la formación superior (Universidad del País Vasco, 2019).

Una segunda dimensión conceptual utilizada en este estudio fue la teoría de Transposición Didáctica de Yves Chevallard (1991), cuyo aporte ha permitido trascender la postura psicologista que caracteriza la didáctica del profesor, para incorporar una visión epistemológica y antropológica del saber enseñando, hecho que se asocia a la "noosfera", como contexto del fenómeno educativo.

La noosfera es el ambiente externo del hecho pedagógico, un lugar de expertos (científicos, políticos, burócratas, gestores y personajes importantes en la vida social y cultural de la nación), los cuales definen la política educativa, la selección de contenidos, los saberes didácticos y las obras de apoyo a la docencia, entre otros aspectos. Situación que genera posteriormente una "transposición externa".

Los postulados e ideas de Chevallard ofrecen una nueva mirada de la didáctica como disciplina pedagógica, donde interesa reconocer la distancia entre el saber experto y el saber enseñado, como motor de desarrollo de una educación de calidad. Al mismo tiempo, introduce una nueva terminología y categorías para comprender la acción pedagógica del docente en el sistema educativo (Villalobos-Claveria \& Melo-Hermosilla, 2019).

En esta perspectiva, Chevallard ayuda a comprender el trabajo docente como una gestión de saberes, donde el saber didáctico es una delimitación epistemológica del saber experto disciplinario, según propósitos formativos, normativa vigente y exigencias institucionales al docente (Chevallard, 1991; Grisales-Franco y González-Agudelo, 2009).

A través de la transposición didáctica se crea un proceso mediante el cual los contenidos y saberes provenientes de un ámbito experto se convierten en saberes a enseñar, contenidos curriculares y actividades de enseñanza. Pero estos saberes que son, a la vez, "objetos de enseñanza", deben ser reconocidos como productos de la cultura disciplinaria y adquieren una naturaleza semejante al saber experto, para luego llegar a ser "objetos de aprendizaje" en los estudiantes: el saber aprendido específico de una profesión (Chevallard, 1991; Grisales-Franco \& González-Agudelo, 2009).

\footnotetext{
1 En original: "We need to systematically reflect on the evidence of learning from our own students, our own classes, and disciplines. We need to build on generic research, but carefully place that within our disciplines. We then need to monitor the success or failure of our efforts to improve our students' learning and then communicate the results of those efforts to our colleagues. The payoff for teaching and learning in this perspective is not research in the traditional sense; it is an orientation activity to be performed in a collegiate way and increasingly together with traditional research within the disciplines". To be erudit professors, scholars must use the same type of thought processes in their teaching, which they apply to their research."
} 
La didáctica del profesor se reconoce en la denominada "transposición interna", que se refiere a los cambios que experimenta el saber a enseñar al convertirse en un saber enseñado. En este espacio, el profesor de aula participa directamente en la elaboración de materiales de aprendizaje, diseño y aplicación de estrategias, como también uso de analogías, ejemplos, ilustraciones, estudios de caso, guías de laboratorios, textos de apoyo, para transformar los saberes científicos en estructuras comprensibles para los estudiantes (Chevallard, 1991; Grisales-Franco \& González-Agudelo, 2009; Verdugo-Perona et al., 2017).

La distancia epistémica entre ambos saberes genera la necesidad de una "vigilancia epistemológica", que procura que el "saber a ser enseñado" sea estructuralmente isomorfo con el "saber sabio", es decir, que ayude a ejercer un control para que el saber que se enseña en la universidad no se desvíe en lo sustancial del saber erudito o científico (Chevallard, 1991).

Cabe advertir que la elección de estas dos perspectivas teóricas (SoTL y Transposición Didáctica) ayudan a describir, explicar e interpretar algunas problemáticas que caracterizan la actual situación de la pedagogía universitaria chilena, específicamente la construcción del saber didáctico del profesor novel.

En esta perspectiva, los objetivos del presente estudio fueron:

1. Analizar el proceso de transferencia didáctica de docentes nóveles en tres universidades regionales chilenas.

2. Caracterizar el proceso de transformación de un investigador (experto disciplinario) en un docente universitario.

Dentro de este ambiente de investigación surgieron diversas interrogantes que problematizan el quehacer didáctico del profesor principiante, tales como:

¿Qué antecedentes biográficos y profesionales tiene un profesor principiante al ingresar a la docencia universitaria?, ¿Cómo se va conformando el conocimiento disciplinar didáctico de un profesor novel?, ¿Cómo este joven profesor enfrenta dificultades o problemas para desarrollar su docencia?, ¿Qué importancia tiene la vivencia de ser docente en la construcción de sus saberes en el ejercicio pedagógico cotidiano?, ¿Cual es la naturaleza del proceso de la transferencia didáctica que efectúa un profesor novel al ejecutar su docencia cotidiana?

Una premisa que se ha aceptado en este estudio es el reconocimiento que este docente joven, en su práctica cotidiana, ha comenzado a construir un saber didáctico adecuado y pertinente a su realidad, pero que todavía no se ha conceptualizado en un modelo de docencia profesional universitario. Este fenómeno ha sido reconocido por SoTL.

\section{Diseño de investigación}

El estudio asume una naturaleza exploratoria y comprensiva sobre la actividad del profesor universitario en cuanto a su ejercicio como docente novel. Se trata de comprender e interpretar la actuación pedagógica de un profesional que se inicia en la docencia universitaria y sus vivencias, por eso asume una mirada global e individual de los sujetos consultados. Situación que ayuda a entender su aplicación en este estudio (Albarello, 2012; Canales, 2016; Vasilachis, 2006).

Esta investigación cualitativa se inscribe en los enfoques fenomenológico, hermenéutico e interpretativo. Al mismo tiempo, asume una matriz metodológica de estudio de casos (Stake, 2005). Bajo esta óptica, cada caso representa una visión singular, donde sus datos son comprendidos profundamente por la visión del investigador enmarcado en su visión epistemológica y conceptual (Albarello, 2012).

Los datos fueron recolectados durante el año 2015 - 2016, en formato de entrevistas en profundidad, con preguntas semiestructuradas y apoyados previamente en un análisis del contenido de su curriculum vitae $(\mathrm{CV})$, sobre una muestra aleatoria de 16 académicos que se desempeñaban en tres universidades chilenas de la región del Biobío. 
Estas tres universidades regionales eran: una, de carácter tradicional y formación católica; otra, una universidad tradicional y laica, y otra, de carácter tradicional y estatal.

TABLA 1

COMPOSICIÓN DE LA MUESTRA

\begin{tabular}{|c|c|c|c|c|c|}
\hline Universidad & Sexo & Profesión & Grado académico & Facultad & Carreras \\
\hline 1 UCSC & $\begin{array}{l}\text { Mujeres: } 5 \\
\text { Hombre: } 1\end{array}$ & $\begin{array}{l}\text { Kinesiólogo, } \\
\text { Licenciado en } \\
\text { Matemática, Bióloga, } \\
\text { Trabajador social, } \\
\text { Nutricionista y } \\
\text { Antropólogo }\end{array}$ & $\begin{array}{l}\text { Licenciado: } \\
\text { Magister: } 4 \\
\text { Doctor: } 2\end{array}$ & $\begin{array}{l}\text { Medicina, Ciencias, } \\
\text { Comunicación y } \\
\text { Ciencias Sociales }\end{array}$ & $\begin{array}{l}\text { Química ambiental, } \\
\text { Kinesiología, Nutrición } \\
\text { y Dietética, Ingeniería } \\
\text { y Trabajo Social }\end{array}$ \\
\hline $2 \mathrm{UdeC}$ & $\begin{array}{l}\text { Mujeres: } 3 \\
\text { Hombre: } 1\end{array}$ & $\begin{array}{l}\text { Abogado, Tecnólogo } \\
\text { Médico, Kinesiólogo, } \\
\text { Periodista }\end{array}$ & $\begin{array}{l}\text { Licenciado:1 } \\
\text { Magister: } 3 \\
\text { Doctor: }\end{array}$ & $\begin{array}{l}\text { Medicina, Ciencias } \\
\text { Sociales y Ciencias } \\
\text { Jurídicas y Sociales }\end{array}$ & $\begin{array}{l}\text { Tecnología Médica, } \\
\text { Periodismo, Derecho, } \\
\text { Kinesiólogo, }\end{array}$ \\
\hline 3 UBB & $\begin{array}{l}\text { Mujer: } 1 \\
\text { Hombres: } 5\end{array}$ & $\begin{array}{l}\text { Fonoaudiólogos (2), } \\
\text { Ingeniero Comercial, } \\
\text { Diseñador Gráfico, } \\
\text { Biólogo Marino y } \\
\text { Psicólogo }\end{array}$ & $\begin{array}{l}\text { Licenciado:1 } \\
\text { Magister:3 } \\
\text { Doctor:2 }\end{array}$ & $\begin{array}{l}\text { Ciencias de la Salud } \\
\text { y de los Alimentos, } \\
\text { Ciencias, Ciencias } \\
\text { Empresariales, } \\
\text { Arquitectura, } \\
\text { Construcción y Diseño }\end{array}$ & $\begin{array}{l}\text { Fonoaudiología, } \\
\text { Ingeniero Comercial, } \\
\text { Diseñador Gráfico, } \\
\text { Pedagogía }\end{array}$ \\
\hline Total: & $\begin{array}{l}\text { Mujeres: } 9 \\
\text { Hombres: } 7\end{array}$ & 14 profesionales & $\begin{array}{l}\text { Licenciado:2 } \\
\text { Magister:12 } \\
\text { Doctor:4 }\end{array}$ & 16 & 12 \\
\hline
\end{tabular}

Fuente: Elaboración propia.

La unidad de análisis es el sujeto profesional que actúa como profesor universitario, independientemente de su campo disciplinario, sexo o edad. Los criterios de inclusión fueron: experiencia menor a 5 años en labores docentes frente a estudiantes de pregrado, jornada completa de trabajo y pertenecer a uno de los ámbitos disciplinarios (Ciencias Sociales, Ciencias de la Salud y Ciencias Experimentales) que fueron agrupados por cada universidad.

Para velar por la fiabilidad y validez de los instrumentos de recogida de datos (entrevista y grilla de análisis del curriculum vitae), el formato de aplicación fue validado por jueces nacionales e internacionales mediante la técnica de fidelidad inter-jueces, que se refiere al grado de acuerdo mostrado en las valoraciones realizadas (Canales, 2016; Vasilachis, 2006). Fueron consultados 6 expertos nacionales en medición (psicólogos y académicos del área de evaluación) y 2 jueces internacionales (estudiantes de doctorado en educación de la Universidad de Barcelona). De esta forma, se buscó mejorar la pertinencia y aplicabilidad de tales herramientas.

Frente a la aplicación de los instrumentos se puede señalar que, una vez seleccionados aleatoriamente los sujetos que cumplían las condiciones de inclusión, se procedió a contactar a un total de 16 docentes de estas universidades regionales. Se solicitó su participación en este proyecto, mediante firma de carta de consentimiento informado (documento que aseguraba el anonimato y confidencialidad de las respuestas). Cabe señalar que dicho documento de naturaleza investigativa contó con la aprobación del Comité de Ética de la Universidad de Concepción. Al mismo tiempo, se tuvo acceso a su respectivo curriculum vitae.

Paralelamente, los integrantes del equipo de investigación (psicóloga, estudiantes de Magister en Pedagogía universitaria y de Educación, entre otros profesionales), procedieron a la aplicación de la entrevista y a una lectura previa del curriculum vitae de los docentes consultados.

Las entrevistas fueron grabadas y posteriormente transcritas en formato Word, para su posterior categorización. Durante la aplicación de dicho instrumento, la entrevistadora generó un ambiente amable, cordial, respetuoso y seguro que estimulase la reflexión y la vivencia de ser un docente universitario. El tiempo utilizado en su aplicación osciló entre 45 minutos y 1 hora, según el carácter y la disposición de cada docente. 
Los resultados de las entrevistas fueron procesados a través del Software Atlas Ti, desarrollándose un análisis de contenido guiado por las categorías previas de la investigación, que fueron consideradas en la construcción de los instrumentos para generar datos y acceder al campo en conjunto con una teoría que sustenta y orienta el estudio (transposición didáctica). No obstante, en el proceso de codificación y categorización de los significados de las acciones y representaciones de los informantes emergieron nuevas categorías (datos inductivos), que se pueden interrelacionar con los datos previos, dando origen a una permanente interacción dialéctica durante todo el proceso de análisis de los datos (Canales, 2016; Vasilachis, 2006).

En cuanto a las grillas de análisis del Curriculum vitae, la información obtenida fue procesada a través del Software Microsoft Excel, con el propósito de obtener mayores precisiones del segmento en estudio y conocer con mayor profundidad elementos asociados a la subjetividad de los profesores nóveles (intereses cognitivos, capacidades y logros que resumen la trayectoria del docente). Este análisis fue orientado por la perspectiva del enfoque SoTL.

\section{Resultados y su interpretación}

Un aspecto importante a destacar es la edad del académico novel. El docente novel suele ser una persona joven, de manera que no existe una diferencia de edad importante entre el profesor y los estudiantes universitarios. Se espera que esta circunstancia sea un factor positivo en tales docentes, pero se sospecha que tal factor tiende a dificultar la adopción de rol docente. Este hecho obtenido de la observación y la experiencia previa de los integrantes del equipo de investigación en pedagogía universitaria, determinó el presente análisis inicial de los datos obtenidos durante la ejecución del estudio.

Cabe señalar que la grilla aplicada al Curriculum Vitae de los docentes fue determinante para descubrir su área o ámbito del desempeño pedagógico en las carreras universitarias, donde ejercían habitualmente su labor docente, dado que existen académicos que imparten cursos básicos de ciencias para diversas carreras, como es el caso de asignaturas de estadística, matemáticas, biología, física, metodología de la investigación, entre otras.

El análisis de los resultados obtenidos fue organizado y agrupado en dos grandes categorías que surgen de los objetivos de la investigación.

\section{El proceso de transferencia didáctica del docente novel}

El estudiante universitario aprende un conjunto de saberes que caracterizan una determinada profesión, pero cuya naturaleza semejante con el saber experto tiende a provocar una "ilusión" gnoseológica en el alumno para apropiarse de esta fuente disciplinaria que forma parte de un determinado campo del saber experto. Por cierto, en esta selección disciplinaria de los contenidos puede ocurrir que el docente no realice esta operación de una forma consciente, por diversas razones y motivos de índole cultural, económica, política o ideológica. Una teoría que ayuda a explicar semejante situación es la Transposición Didáctica de Chevallard (1991).

Desde el punto de vista del conocimiento, el profesor universitario novel tiende a utilizar su saber experto en la docencia de pregrado, pero deja su investigación disciplinaria para la docencia de postgrado, es decir, este profesor tiende a reproducir un patrón de formación que ha vivenciado durante su trayectoria y lo aplica en la formación de sus estudiantes. Así, por ejemplo, tiende a utilizar los mismos libros o manuales de estudio, las guías de laboratorio, ejemplos que han sido validados por sí mismos o por sus colegas, entre otras situaciones. Algunos fragmentos ilustrativos de dicha tendencia se exponen a continuación. 
Los avances científicos son demasiado rápidos o profundos como para que el alumno, en las condiciones actuales, pueda entenderlas; hay programas de bioinformática que no les puedo enseñar ahora. No sabría manejar la teoría que está bajo esos programas informáticos, por ejemplo. Eso tiene que ser enseñado en un curso de postgrado. Entonces el avance científico no lo puedo explicar, porque tengo que enseñarles lo básico primero y no alcanzo a enseñar lo actual, no lo puedo hacer, tiene que hacer cursos de postgrado para eso. (biólogo, Cs. de la Salud, U3).

En este momento yo no estoy relacionando mucho la docencia con la investigación, porque el proyecto que estoy desarrollando es bastante complejo para estos alumnos. Pero ahora pretendo enviar proyectos de investigación para fondos concursables y ahí sí tengo interés en acercarme a estos alumnos y crear un nexo, tanto en el pregrado, como en el postgrado. (kinesióloga, Cs. de la Salud, U2)

En este último caso se reconoce que la docente debe crear una instancia para incorporar a sus estudiantes en la investigación disciplinaria, pues advierte la distancia entre un tipo de saber experto y otro de naturaleza didáctica y contextualizada a su área de formación profesional. Interesante aspecto que se puede destacar e interpretar de acuerdo a los postulados de la transposición didáctica y al desafío asociado a la vigilancia epistemológica. Este último proceso destaca que el saber a ser enseñado debe ser estructuralmente isomorfo con el saber sabio, es decir que ayude a ejercer un control epistémico para que el saber que se enseña en la universidad no se desvíe en lo sustancial del saber erudito o científico (Chevallard, 1991).

El identificar la obsolescencia del conocimiento presente en los planes y programas de estudio es otra cuestión que se reconoce en las expresiones de los docentes nóveles. Hay una preocupación por el avance del conocimiento científico, las nuevas creaciones del mercado y los productos que surgen en el área disciplinaria. En esta perspectiva, los nuevos profesores señalan:

Encuentro muy obsoletos los programas, la lista de contenidos y la bibliografía. Son los mismos libros que yo leía cuando era estudiante. Los contenidos del programa son contenidos que ya no están en el mercado, pues en el mercado ya están hablando de otros términos. Yo creo que hay que actualizar un poco la información, al menos en el Diseño que es una disciplina que está constantemente cambiando. Hoy día se está necesitando que el estudiante de Diseño esté vinculado más a internet, todo está mutando hacia allá, las estrategias de marketing se están haciendo por internet, y se requiere formar profesionales que manejen las redes sociales y posicionen las marcas o la publicidad a través de dichas redes. (diseñador, Cs. Sociales, U3).

Al planificar, cuando yo llegué acá, modifiqué completamente esta asignatura, porque los estudiantes estaban aprendiendo a utilizar "Flash". Este es un programa que ya no se utiliza en ninguna parte del mundo. No creo que, en una universidad, a los alumnos se les deba enseñar Flash, porque solamente es una herramienta. Entonces, lo que se hizo fue modificar la asignatura, para enseñar a realizar propuestas visuales en software de diseño visual. (hombre, periodista, Cs. Sociales, U2).

Si se acepta que el docente universitario es un investigador de frontera del conocimiento, entonces esta preocupación docente se manifiesta al realizar una mirada externa (desde la noosfera como lugar epistémico) a los contenidos disciplinarios del programa de estudio de una asignatura, con lo cual se "vigila" la actualidad de dicho contenido en la formación profesional. Situación que ayuda a evitar su obsolescencia ("envejecimiento biológico y moral”) y se relaciona con la dimensión de vigilancia epistemológica, asociada a la transposición didáctica (Chevallard, 1991).

La vinculación con la "noosfera" y la universidad es un ámbito interesante de conocer, pues permite comprender la actitud que asume este profesor novel cuando inicia su trabajo docente. En 
general, vemos que los docentes consultados tienden a adaptarse a la normativa y exigencias laborales que plantean sus respectivas casas de estudio; situación que se muestra en la organización de su docencia, la planificación de clases y los requisitos evaluativos que los profesores jóvenes plantean en sus cátedras. De este modo, los docentes revisan el programa, elaboran el syllabus de sus asignaturas, promueven un trabajo en equipo, consultan a colegas o a jefes de carrera que ayuden a dimensionar la futura labor pedagógica que deberá ejecutar. Algunas de dichas situaciones se exponen a continuación.

Generalmente cada año planificamos la asignatura en conjunto con otro profesor. Tomamos como referencia el calendario que nos envían, hacemos una reunión con los profesores del año anterior, revisamos el tema de las evaluaciones, encontramos una pauta en común, una rúbrica y hacemos la carta Gantt que después la entregamos a la dirección. Este es nuestro modus operandi, que no es muy distinto a todas las otras carreras. (diseñador, Cs. Sociales, U3).

La secuencia de contenidos viene dada por el programa y eso lo conversamos con todos los docentes del departamento; se revisa este programa, cada cierto tiempo, y la duración de cuanto voy a asignar yo a cada contenido (que depende de los casos jurídicos que he tenido), que se refleja en mi Syllabus, así lo voy organizando. Generalmente, las lecturas complementarias las dejo para el final. (hombre, abogado, Cs. Sociales, U2).

Bueno, lo primero es el tema del Syllabus, porque en las asignaturas modulares, el profesor a cargo de la asignatura nos solicita hacerlo. Antes eran los contenidos, ahora nos hemos centrado en el tema de los resultados de aprendizaje (problema que surge de la labor profesional). Nos cuestionamos acerca de qué necesita el alumno a ese nivel. Si veo que hay algunas falencias, trato de integrar en mis clases algunos conceptos previos que yo considero necesarios. Entonces, la idea es planificar el tema de los contenidos, pero centrándome en las actividades prácticas para el laboratorio. (kinesiólogo, Cs. de la Salud, U2).

Frente a dificultades o problemas para desarrollar su docencia, el docente novel tiende a recurrir a sus colegas con más trayectoria y no necesariamente a expertos o pedagogos. Con sus colegas comparte experiencias sobre las dificultades que se viven, los problemas con los alumnos y las formas más apropiadas para resolver dichas situaciones inusuales. Así, por ejemplo, se tiende a integrar conocimiento disciplinar de diferentes años de la carrera.

Nosotros estamos trabajando con la "Wiki", y simulamos en la asignatura Intervención, modelos micro de intervención con estudiantes de la misma carrera. Por ejemplo, nuestros estudiantes de cuarto año están aprendiendo qué es la terapia vocal y los estudiantes de tercer año están cursando la asignatura de Eufonía, adquiriendo una técnica vocal. Entonces, lo que hicimos fue asociarnos con la colega de Eufonía, donde mis estudiantes de cuarto son tutores de los estudiantes de tercero, son sus pacientes, son sus usuarios. Todas las semanas suben videos a la plataforma, acerca de cómo es la intervención, y los evalúo a través de una rúbrica, que ellos conocen desde el inicio de la asignatura. Se les evalúa por medio de la Wiki, porque para nosotros también es complejo ir a evaluar cada vez que se junte con su usuario, entonces ellos editan los videos y los suben. (fonoaudiólogo, Cs. de la Salud, U3).

En este juego de reconstrucción, los docentes interpretan y filtran las materias de enseñanza con una intención didáctica, explorando nuevos lenguajes y recursos más asequibles a las estructuras de conocimiento de los estudiantes. Por cierto, el profesor novel no es consciente que hace una transferencia didáctica, pues acepta o afirma que enseña la disciplina tal como le fue enseñada en su formación, ya sea en pregrado, como en postgrado. Sus maestros son un referente para abordar su trayectoria pedagógica, sobre todo cuando ejecutan labores docentes. 
Yo básicamente hago las clases basado en lo que me enseñaron los maestros que tuve en mi época de estudiante de pregrado y de postgrado, hice magister, hice doctorado, hice postdoctorado, y el estilo de las clases es más o menos el estilo de las clases que hacen esos maestros, yo aprendí y creo que está bien. A mí me satisface, al menos, ese tipo de enseñanza, es lo que conozco. (biólogo, Cs. de la Salud, U3).

Nosotros tenemos una formación que no es de pedagogía, pero estamos, de alguna forma, vinculados con la enseñanza. El fonoaudiólogo trabaja en escuelas de lenguaje, trabaja enseñando a sus usuarios. Cuando yo llegué a la universidad no hubo algo como "yo voy a hacer clases, cuáles son las técnicas", no tenía idea de nada. Yo estaba feliz de estar en la universidad. .. uno tiene una percepción de sus profesores de la universidad, que tampoco tuvieron mucha pedagogía con uno, entonces uno cree que es llegar y pararse adelante y empezar a hablar, y después se va encontrando con que es mucho más complejo y mira ... el lío en el que te metiste. (fonoaudiólogo, Cs. de la Salud, U3).

Un aspecto en el cuál concuerdan los profesores nóveles es la elaboración de guías didácticas para sus cursos. Guías que son propuestas, en el caso de algunos docentes, bajo sus propias creencias y expectativas acerca del potencial aprendizaje de los alumnos. Al mismo tiempo, otros docentes suelen seguir las indicaciones dadas por la Dirección de Docencia para la elaboración de estos recursos de enseñanza.

Cuando terminé mis estudios de doctorado me fui a trabajar a una universidad privada,... Ahí me enfrenté con el trabajo de estar en un aula, nunca había hecho clases más allá de lo que hace uno como estudiante. Yo pasé de nunca haber hecho clases y tomé diez asignaturas inmediatamente el primer año que tuve de trabajo. Partí como "profesor taxi”, hacía clases en cinco universidades, corría de una a otra, y con cero de experiencia previa; tampoco había tomado cursos de didáctica, ni de pedagogía. En mi caso tuve un aprendizaje en terreno, tampoco me encargué de investigar. Más allá de lo que uno aprende como psicólogo, como de los procesos de aprendizajes generales, pero sin didáctica o pedagogia. (psicólogo, Cs. Sociales, U3).

Yo hacía clases expositivas, pero este último tiempo he dejado de hacerlo y hago algo similar a TBL (Team Based Learning o Aprendizaje basado en Equipos). Se le entrega un documento al alumno antes de la clase, él lo lee y después desarrollamos una guía respecto de eso, de sus dudas. Aparte de eso tengo muchos laboratorios, hago un laboratorio, luego clases y, además, tengo una gran dedicación a investigación. Esa es mi actividad docente general... (kinesióloga, Cs. de la Salud, U2).

A pesar de provenir de diferentes campos disciplinarios, este profesor novel tiene una actitud semejante para emprender este desafío, pues carece de herramientas pedagógicas y didácticas para iniciar el desarrollo del proceso docente. Se reconoce una actitud científica en enfrentar los problemas que les provoca su propia docencia, aun cuando a veces sea un acercamiento tentativo y provisorio en la búsqueda de una docencia adecuada a su propia realidad, cuyos resultados serán motivo de reflexión y análisis. Este resultado concuerda con los planteamientos del enfoque SoTL, sobre la construcción del conocimiento pedagógico.

\section{Transformación de un investigador en docente universitario}

El criterio de llegar a ser un buen docente es tener un buen dominio de la disciplina, del saber experto (Guerra y Montenegro, 2017; Hutchings y Shulman, 1999; Shulman, 1987). Sin embargo, también se reconocen voces que manifiestan su preocupación por la forma en que se comprende la pedagogía universitaria. 
Yo trato de enseñarles lo más que puedo, básicamente, sigo el sistema aristotélico del quehacer académico, que es que tú tienes que tratar de traspasar todo tu conocimiento al alumno, y si alguna vez se cumple que el alumno sea mejor que el maestro, ahí estamos formando académicos. Esto es lo que yo quiero inculcar en mis alunos. (biólogo, Cs. de la Salud, U3).

A mí me asusta cada vez más que la universidad está tendiendo a modelos de syllabus, donde al docente le pasan el programa, la planificación, la metodología y los contenidos de lo que tienes que decir. Esto es un control y una universalización de la formación, que va en contra de lo que antes llamaban la libertad de cátedra. Para innovar, para ser profesor se debe tener un equilibrio y no estar sujeto a estas estructuras tan rígidas, por eso creo que para cambiar es bueno tener asignaturas más colegiadas, porque así en equipo vamos entre todos aportando y también es más entretenido. No es tan solo tu responsabilidad como profesor, que éste sea tu ramo, somos parte de un proceso continuo y no como es ahora, que es muy fragmentado. (psicólogo, Cs. Sociales, U3).

Cabe señalar que la participación en labores de gestión de la enseñanza, el trabajo colegiado en las carreras, la pertenencia a comunidades docentes, brindan la oportunidad para que los docentes nóveles puedan desarrollar en forma progresiva un conocimiento pedagógico situacional en consonancia con el escenario científico e institucional en el cual se desenvuelven (Feixas, 2002; Rodríguez Torres et al., 2020; Shulman, 1987). Situación vivenciada en el quehacer cotidiano que impulsa a ser un mejor docente, como expresión de su compromiso y responsabilidad profesional de ser un profesor universitario. Desafío que genera otra categoría de análisis en estos resultados, la cual se expone a continuación.

\section{a) Detección de necesidades formativas y auto-exploración.}

Una vez que estos profesionales se transforman en docentes universitarios, les surgen inquietudes por conocer sobre la naturaleza de la pedagogía y, al mismo tiempo, tienden a comparar la formación recibida con el desempeño que ellos tienen frente a sus estudiantes (Bozu, 2009). En este análisis crítico y comparativo se evalúa tanto la enseñanza, como el aprendizaje. Particularmente importante es la forma de evaluación que utilizan para calificar a sus estudiantes. Aquí surgen incertezas y dificultades para lograr una gestión evaluativa de sus cursos, más allá de lo que propone la respectiva Dirección de Docencia (unidad encargada de los procesos administrativos y docentes).

Por eso, resulta ser muy importante asumir la corriente internacional denominada "Enfoque Académico de la Enseñanza y el Aprendizaje" (SoTL), cuyo enfoque ha permitido construir esta categoría. En este sentido, se busca comprender la forma que asumen los procesos asociados a la enseñanza-aprendizaje en la docencia universitaria y la función que asume el docente novel, para promover una educación de calidad. Así, por ejemplo, esta categoría se ilustra con el siguiente fragmento narrativo de un docente consultado.

Básicamente en todos los cursos que hago les digo a mis alumnos que tienen que saber lo que es un paradigma, una inferencia, una deducción. Que tienen que saber lo que es una teoría, qué es una hipótesis, una ley, saber lo que es una emisión, porque ninguno de ellos es lo mismo .... Entonces en ciencia básicamente tienen que saber eso, los estimulo a estudiar quienes han sido los filósofos de la ciencia, por ejemplo, Thomas Kuhn, Karl Popper, Lakatos. A mi entender, ellos tienen que saber cómo se mueve la ciencia a través de los siglos, y cómo nosotros trabajamos haciendo ciencia. Los investigadores trabajamos bajo la filosofía científica de Popper, nuestra forma de hacer ciencia es popperiana. Hay otros filósofos de la ciencia, como Kuhn, pero nosotros no trabajamos a Kuhn. A los alumnos, generalmente a las personas que están terminando acá, les digo lo que tienen que saber. Ellos nunca habían escuchado acerca de Kuhn, y están haciendo una tesis de laboratorio. ¡Eso yo lo encuentro increíble! (biólogo, Cs. de la Salud, U3). 
A través de esta lectura se puede inferir la interrelación que surge entre la perspectiva disciplinaria del docente y el potencial desarrollo de la investigación pedagógica que se puede aplicar en el aula. Idea expuesta por el enfoque de SoTL en el contexto de una transformación de la pedagogía universitaria, al permitir una mayor profesionalización del académico en temas de pedagogía.

La interpretación de las entrevistas aplicadas a docentes nóveles permite reconocer una intención de incorporar la función docente en su quehacer académico. Hay una preocupación por seguir la normativa universitaria, buscando responder a los desafíos que conlleva la formación profesional superior. Muchas de sus actividades pueden ser comprendidas dentro de la teoría de la Transposición Didáctica. Por cierto, en este proceso de crecimiento profesional surge la necesidad de auto-perfeccionamiento y capacitación que facilite y potencie su propio desarrollo académico; necesidad que hasta la fecha es una tarea pendiente, a pesar de los esfuerzos de las instituciones universitarias.

Finalmente, cabe destacar que no se encontraron diferencias entre los docentes novéles de estas casas de estudios superiores en cuanto a los aspectos consultados; pues se sospecha que, al estar dirigidos por la aplicación del enfoque de competencias promovida por el Ministerio de Educación de Chile, su docencia tiende a repetir estos lineamientos que enmarcan su quehacer pedagógico cotidiano.

\section{A modo de teorización de los resultados y una discusión crítica}

La lectura de los resultados del estudio permitió identificar aspectos claves para cartografiar el proceso de construcción del saber pedagógico en docentes novéles, es decir, mediante un razonamiento inductivo se ha procedido a teorizar estos hallazgos (Rodríguez Torres et al., 2020). Por ello, los docentes consultados se pueden agrupar en tres modos de construcción del saber didáctico, los cuales se explicitan a continuación.

a. Formación básica y socialización profesional. Este primer modo se refiere al área del conocimiento caracterizada por la formación inicial del docente, como en su disciplina de especialización (estudios de pregrado y postgrado). En esta etapa, el profesor novel comienza a adquirir y a elaborar un conocimiento científico que, posteriormente, empleará en su enseñanza, con las adaptaciones necesarias para tales efectos. Su participación en espacios formativos posteriores, tales como defensa de tesis doctoral, asistencia a congresos, seminarios, pasantías, le brindan la posibilidad para adquirir conocimientos y habilidades claves, tanto para la función investigadora, como para la función de enseñanza.

El profesor novel comienza a pensar la docencia desde su propia disciplina y los desafíos que debe enfrentar lo hace pensando desde este mismo campo disciplinario. Es importante mencionar que los profesores nóveles descubren la afinidad por la docencia, como consecuencia de la especialización en su disciplina (o área de investigación) y no en el sentido opuesto.

Los docentes indican que se inician en un proceso gradual, primero en ayudantías, luego con tutorías de laboratorios, hasta llegar a ser profesores con mayor autonomía (todo en el marco de su formación académica y laboral), siendo la docencia un aspecto siempre latente en su plano laboral, que facilita la movilización de los saberes pedagógicos adquiridos de forma consciente. Sin embargo, la complejidad del acto pedagógico los impulsa a buscar nuevas teorías o modos de ejercer docencia, ya sea mediante capacitación en sus casas de estudio o la posibilidad de acceder a estudios de postgrado en el campo de ciencias de la educación. Situación verificada en el examen del Curriculum Vitae.

b. Inducción profesional y socialización en la práctica. Una segunda manera de enfrentar esta etapa inicial se reconoce cuando el profesor principiante alude a la formación recibida en un programa doctoral, para llegar a ser un buen docente.

$\mathrm{Al}$ igual que en la fase anterior, ésta es otra forma de entender sus primeros años de actividad 
profesional como docente universitario, una etapa en la cual se produce una socialización académica, tanto en los ámbitos de la enseñanza, como de la investigación (Bozu, 2009). Espacio donde el profesor novel enfrenta un proceso de auto-inducción profesional, caracterizado por un ajuste y adaptación a los procedimientos institucionales que posee su casa de estudios superiores (Porto Castro \& Mosteiro García, 2016). En este contexto, la socialización del docente se puede manifestar en tres ámbitos:

i. La sala de clase como espacio para la docencia. El tipo de asignatura, la carrera y el tipo de alumno suelen ser factores que inciden en esta socialización inicial del docente principiante.

ii. El contexto de la carrera o programa de formación donde ejerce, es otro espacio en el cual el docente novel internaliza elementos de la cultura organizacional universitaria, ya sea en la relación con sus colegas, las reuniones de departamento, la elaboración de programas y syllabus, la participación en la aplicación de sistemas de evaluación, la valoración que posee la investigación en la docencia, entre otros.

iii. El contexto sociocultural de la casa de estudios, donde el docente novel debe responder a desafíos relacionados con el rol social que posee dicha institución en el medio de sus estudiantes, así como el tipo de orientación formativa o modelo de formación profesional que ofrece al estudiantado y que se reconoce como modelo educativo.

En suma, estos diferentes espacios a través de los cuales transita un docente novel constituyen oportunidades para construir su propio conocimiento didáctico (Hutchings \& Shulman, 1999; Rodríguez Torres et al., 2020). Cabe señalar que dicho docente, por su falta de experiencia, no tiene todavía un conocimiento tácito del quehacer pedagógico y, por ende, aún no ha ritualizado su saber didáctico (Chocarro de Luis et al., 2013).

c. El Perfeccionamiento como politica institucional de la universidad. Este es otro espacio formativo para un docente principiante. A través de sucesivas capacitaciones se espera disponer de un profesor preparado para aplicar nuevas reformas y/o políticas institucionales que se exigen a las casas de estudios superiores. A pesar de ser impulsadas por la autoridad competente, no satisfacen las expectativas del docente novel, quien tiende a recurrir a sus colegas con mayor experiencia y trayectoria académica.

Este es un gran desafío institucional para las universidades participantes, llegar a ofrecer un perfeccionamiento pertinente, adecuado y válido a la docencia universitaria que, al mismo tiempo, sea global y específico en cada campo profesional (Guzmán, 2012).

En otras palabras, se trata de ir más allá de la transmisión de conocimientos que hace el profesor frente a sus alumnos, para promover una reflexión sobre la acción de aprender qué sucede cuando el estudiante tiene autonomía para pensar (Albergaria-Almeida, 2010; Medina \& Jarauta, 2013; Verdugo-Perona et al., 2017). En este escenario, el profesor principiante comienza a construir su propia trayectoria docente, mediante una actividad investigadora que se promueve en las universidades para su desarrollo académico. Surgen necesidades formativas del propio docente cuando inicia su carrera académica, especialmente de los docentes nóveles. Resultado que se vincula al enfoque SoTL.

Por último, frente a los nuevos desafíos didácticos que enfrenta el profesorado universitario surge la búsqueda de experiencias exitosas, la elaboración de perfiles académicos basados en competencias, concursos y programas de investigación sobre pedagogía universitaria, elaboración de material didáctico de apoyo al aprendizaje universitario, nuevas formas de evaluación alternativas, entre otras iniciativas tendientes a formular un modelo innovador en docencia profesional superior (García-Prieto et al., 2019).

Por cierto, esta nueva modalidad de la visión didáctica del profesor novel demanda un modelo de formación docente que las instituciones de educación superior deben conceptualizar y aplicar en el desarrollo académico de sus docentes. Tarea pendiente y en pleno desarrollo. 


\section{A modo de conclusiones}

De los resultados obtenidos en este estudio es posible obtener las siguientes conclusiones:

1. En la docencia de profesores nóveles, se puede reconocer el enfoque de la Transposición Didáctica y sus mecanismos asociados, pero sin tener mayor conciencia ni comprensión de dichos procedimientos pedagógicos. Tal vez sea por desconocimiento de dicha teoría. Al mismo tiempo, el enfoque denominado "Scholarship of Teaching and Learning" (SoTL) sirve para explicar la investigación en didáctica que realizan los docentes nóveles, pues el dominio de la investigación científica y la comprensión de sus etapas permite que sea aplicada a la docencia universitaria, donde sus diversas problemáticas son abordadas desde la ciencia y el razonamiento analítico que la caracteriza.

2. En relación con el proceso de transformación de un investigador (experto disciplinario) en profesor universitario, el docente novel se desenvuelve en diferentes contextos y asume distintos modos, ya expuestos en la teorización de resultados, que permiten concluir que la construcción del conocimiento didáctico constituye un verdadero desafío intelectual para construir su propio saber pedagógico. En esta perspectiva, la acción pedagógica de los docentes es el resultado de un proceso sociohistórico en donde los modelos de sus profesores (contexto de su formación académica), son determinantes en este aspecto, en la medida que son un referente más cercano frente a las situaciones propias del ejercicio de la docencia. Además, la concordancia entre la formación académica y la docencia se complementan dentro de un ambiente común que determina la misma carrera.

Finalmente, cabe afirmar que el conocimiento y la oportunidad de reconocer la opinión de los profesores nóveles acerca de este "desconocido" proceso de transferencia didáctica, así como el camino de su transformación en docentes, resulta ser de gran interés para avanzar hacia programas de mejoramiento institucional que incentiven la generación de verdaderas comunidades profesionales, en consonancia con los desafíos académicos de esta sociedad del conocimiento.

\section{Agradecimientos}

Estudio financiado por Proyecto de investigación: Fondecyt n 1130803 - "El proceso de transferencia didáctica del docente universitario no pedagogo en la formación profesional superior. Un estudio de caso".

\section{Referencias}

Albarello, L. (2012). Apprendre à chercher. L'acteur social et la recherche scientifique. De Boeck \& Larcier S.A.

Albergaria-Almeida, P. (2010). Scholarship of teaching and learning: An overview. Journal of the World Universities Forum, 3(2), 143-154.

Bolívar, A., \& Caballero, K. (2015). El profesorado universitario como docente: Hacia una identidad profesional que integre docencia e investigación. REDU. Revista de Docencia Universitaria, 13(1), 57-77. https://doi.org/10.4995/redu.2015.6446

Bozu, Z. (2009). El profesorado universitario novel y su proceso de inducción profesional. Magis, Revista Internacional de Investigación en Educación, 1(2), 317-328.

Brunner, J. J. (2015). Medio siglo de transformaciones de la educación superior chilena: Un estado del arte. In A. Bernasconi (Ed.), La educación superior de Chile: Transformación, desarrollo y crisis (pp. 21-107). Ediciones U.C.

Canales, M. (2016). Metodologías de investigación social: Introducción a los oficios. LOM.

Chevallard, Y. (1991). La transposition didactique: Du savoir savant au savoir enseigné. La Pensée sauvage. 
Chocarro de Luis, E., Sobrino Morrás, Á., \& González-Torres, M. del C. (2013). Scholarship of Teaching and Learning: Un modelo de desarrollo profesional de los profesores universitarios. Revista Electrónica Interuniversitaria de Formación del Profesorado, 16(11), 5-14. https://doi.org/10.6018/reifop.413601

Fanghanel, J., Pritchard, J., Potter, K., \& Wisker, G. (2016). Defining and supporting the Scholarship of Teaching and Learning (SoTL): A sector-wide syudy. Higher Education Academy. https:// repository.uwl.ac.uk/id/eprint/2066/1/literature_review.pdf

Feixas, M. (2002). El profesorado novel: estudio de su problemática en la Universidad Autónoma de Barcelona. Boletín de la RED-U, 2(1), 33-44.

Fernández Lamarra, N., \& Pérez Centeno, C. (2011). La profesión académica universitaria en América Latina, en perspectiva comparada. Educação: Revista do Centro de Educação, 36(3), 351-363. http://dx.doi.org/10.5902/19846444

García-Prieto, F., Pozuelos-Estrada, F., \& Álvarez-Álvarez, C. (2019). La evaluación de aprendizajes del alumnado por parte del profesorado universitario novel. Formación Universitaria, 12(2), 3-16. https://dx.doi.org/10.4067/S0718-50062019000200003

Grisales-Franco, L., \& González-Agudelo, E. (2009). El saber sabio y el saber enseñado: Un problema para la didáctica universitaria. Educación y Educadores, 12(2), 77-86. https://doi.org/10.5294/edu.2020.23.1

Guerra, P., \& Montenegro, H. (2017). Conocimiento pedagógico: Explorando nuevas aproximaciones. Educação e Pesquisa, 43(3), 663-680. http://dx.doi.org/10.1590/S1517-9702201702156031

Guzmán, C. (2012). Enseñanza reflexiva y profesores universitarios nóveles: Desafíos para la mejora de la enseñanza. Revista de la Educación Superior, 3(163), 115-137. https://doi.org/10.36857/resu.2020.193

Hutchings, P., \& Shulman, L. (1999). The scholarship of teaching: New elaborations, new developments change. The Magazine of Higher Learning, 31(5), 10-15.

https://doi.org/10.1080/00091380009601717

Insaurralde, M. (2016). Enseñar en las universidades y en los institutos de formación docente (Colección Desarrollo). Noveduc.

Medina, J. L., \& Jarauta, B. (2013) Enseñanza y Aprendizaje en la educación superior. Síntesis S.A.

Porto Castro, A., \& Mosteiro García, M. (2016). Calidad y formación del profesorado novel en las universidades públicas gallegas. Panorama, 10(19), 8-17. https://doi.org/10.15765/pnrm.v10i19.830

Rodríguez Torres, Á., Rodríguez Alvear, J., \& Arias Moreno, E. (2020). El profesorado universitario novel en schock: Propuestas de mejora. Revisión sistemática. Revista Educare, 24(1), 245-269.

Shulman, L. (1987). Knowledge and teaching: Foundations of the new reform. Harward Educational Review, 57(1), 1-22.

Stake, R. (2005). Investigación con estudios de caso. Morata.

Tardif, M. (2004). Los saberes del docente y su desarrollo profesional. Narcea.

Tuning América Latina. (2007). El proyecto Alfa Tuning América Latina, 2004-2007. http://tuning. unideusto.org/tuningal/

Universidad del País Vasco. EuroSoTL. (2019, June 13-14). Explorando nuevos campos a través de la enseñanza y el aprendizaje. Anais do 3 Congreso Europeo de Scholarship of Teaching and Learning. Bilbao,

España. https://www.ehu.eus/es/web/eurosotl-2019/home

Vasilachis, I. (2006). La investigación cualitativa. Gedisa.

Verdugo-Perona, J., Solaz-Portolés, J., \& Sanjosé López, V. (2017). El conocimiento didáctico del contenido en ciencias: Estado de la cuestión. Cadernos de Pesquisa, 47(164), 586- 611. https://doi.org/10.1590/198053143915.

Villalobos-Claveria, A., \& Melo-Hermosilla, Y. (2019). Narrativas docentes como recurso para la comprensión de la transferencia didáctica del profesor universitario. Formación Universitaria, 12(1), 121-132. http://dx.doi.org/10.4067/S0718-50062019000100121

Walker, V. (2016). El trabajo docente en la universidad: Condiciones, dimensiones y tensiones. Perfiles educativos, 38(153), 105-119. https://doi.org/10.22201/iisue.24486167e.2020.168 


\section{Nota sobre la autoría}

Alejandro Villalobos-Claveria: definición del contenido intelectual del manuscrito; redacción y argumentación de la introducción del artículo; elaboración de marco teórico; diseño investigativo; análisis y teorización de resultados; definición de contenido intelectual de la discusión del artículo; elaboración de conclusiones a partir de la combinación e integración de resultados obtenidos. Yenia Melo-Hermosilla: Redacción de resumen; organización de datos para su análisis; análisis de resultados; definición de contenido intelectual de la discusión del artículo (argumentos bibliográficos); elaboración de conclusiones a partir de la integración de resultados obtenidos; análisis bibliográfico e incorporación de bibliografia; revisión del manuscrito para dejarlo de forma aceptable para publicación.

\section{Disponibilidad de datos}

Los datos no pueden ponerse a disposición del público, por la sensibilidad de la información contenida que está reglamentada por la ley $\mathrm{N}^{\circ} 19.628$ de Chile, que regula el trato de los datos de carácter personal.

\section{Cómo citar este artículo}

Villalobos-Claveria, A. A., \& Melo-Hermosilla, Y. M. (2021). Desafíos didácticos del saber pedagógico del docente universitario novel chileno. Cadernos de Pesquisa, 51, Artículo e07820. https://doi.org/10.1590/198053147820 